\title{
ESPINACA FRESCA, SUPERCONGELADA Y EN CONSERVA: CONTENIDO DE VITAMINA C PRE Y POST COCCIÓN
}

\section{FRESH, FROZEN AND CANNED SPINACH: VITAMIN C CONTENT BEFORE AND AFTER COOKING}

\author{
Andrés Fabián Pighín G., Ana Lía Rossi de R. \\ Universidad Nacional de Luján. Departamento de Ciencias Básicas, Buenos Aires, Argentina.
}

\begin{abstract}
Vitamin C stability, new variables of cultivation, management and post harvest conservation of vegetables and culinary treatments previous to consumption can produce modifications in the content of this vitamin. To obtain actualized information of vitamin $C$ content in fresh and processed food, this nutrient was determined in spinach in the fresh state and after subjected to current methods of conservation. Vitamin C determination by HPLC included ascorbic and dehydroascorbic acid. Mean values of vitamin $C$ (mean $\pm S D)$ in spinach were: fresh, $44.0 \pm 8,74$ $\mathrm{mg} / 100 \mathrm{~g}$; quick frozen, $24,2 \pm 14.81 \mathrm{mg} / 100 \mathrm{~g}$; and canned, $25.0 \pm 1.9 \mathrm{mg} / 100 \mathrm{~g}$. This variability depends of genotypic factors and conservation conditions. Microwave and steam cooking generated the most retention of the nutrient, while boiling generated great losses of both vitamin forms.
\end{abstract}

Key words: Spinach; vitamin C; ascorbic acid; dehydroascorbic acid; HPLC.

Este trabajo fue recibido el 21 de julio de 2009 y aceptado para ser publicado el 7 de Abril de 2010.

\section{INTRODUCCIÓN}

La vitamina $\mathrm{C}$ es un nutriente indispensable en la dieta de los seres humanos. Su función más difundida esta relacionada con su poder antioxidante, que le permite desactivar una gran variedad de especies reactivas de oxígeno y nitrógeno en sistemas acuosos. Además actúa como cofactor de enzimas que participan en la síntesis de colágeno, carnitina y neurotransmisores. También se ha demostrado que estimula al sistema inmune, tiene efectos beneficiosos en pacientes con determinados tipos de lesiones cancerosas o precancerosas y facilita la absorción intestinal de hierro no hemínico de los alimentos. En los vegetales es parte del sistema de defensas contra el stress fotooxidativo, entre otras funciones. (1 - 4).

Está compuesta por dos formas activas: el ácido ascórbico (AA), que por oxidación enzimática (ascórbico oxidasa) y química genera el ácido dehidroascórbico (ADHA). Este último es también susceptible a la oxidación a ácido dicetogulónico que carece de actividad vitamínica.

El contenido de vitamina $\mathrm{C}$ en vegetales resulta ser muy variable tanto por causas genotípicas como de manejo pre y poscosecha (5). En los tratamientos de conservación poscosecha, la pérdida de vitamina depende fundamentalmente del tiempo y la temperatura de almacenamiento, de la exposición a la luz y del grado de daño tisular. (6 - 8).

La espinaca (Spinacea oleracea L y sus variedades) es un alimento bajo en calorías, con bajo contenido de grasas, relativamente bajo en proteínas y buen aportador de fibra y micronutrientes como vitamina $\mathrm{C}$, vitamina $\mathrm{A}$ y minerales, especialmente hierro. (5 - 9).

La espinaca fresca puede cosecharse en los meses de invierno y primavera, su cultivo resiste bajas temperaturas aunque con menor rendimiento y es perjudicada por las lluvias intensas. Es altamente perecedera con pérdida de características nutricionales y sensoriales (color, flavour y textura) que afecta su aceptabilidad por el consumidor. Favell (9) demostró que por la susceptibilidad a la oxidación de la vitamina $\mathrm{C}$, su contenido resulta un indicador sensible y adecuado de una buena conservación del vegetal durante el procesamiento, transporte y almacenamiento.

Durante todo el año puede accederse a espinaca 
conservada industrialmente por congelación rápida a $-18{ }^{\circ} \mathrm{C}$ (supercongeladas) o en conserva. Los tratamientos industriales destinados a prolongar el tiempo de conservación de la espinaca, como el supercongelado disminuyen el contenido de este nutriente. Favell (9) demostró que la pérdida de actividad vitamínica en vegetales congelados ocurre principalmente durante el blanqueo necesario para inactivar las enzimas. También pueden esperarse pérdidas importantes posteriores, que son favorecidas por condiciones no isotérmicas tanto durante el transporte y almacenamiento en exhibidores comerciales y en freezer doméstico. Además es razonable esperar una pérdida del contenido de vitamina $\mathrm{C}$ en la elaboración de los vegetales en conserva que incluye el escaldado o blanqueo de las hojas y esterilización industrial del recipiente herméticamente cerrado, aunque no se cuenta con evidencias experimentales al respecto.

Somsub y colaboradores (2) estudiaron el efecto de los métodos tradicionales de cocción aplicados en alimentos vegetales de Thailandia y concluyeron que hay una importante pérdida del contenido de vitamina $\mathrm{C}$ en los mismos. El hervor disminuye significativamente el contenido de vitamina $\mathrm{C}$ y la magnitud depende principalmente de su marcada solubilidad en agua y la elevada temperatura aplicada que favorecen su degradación.

Actualmente la información disponible en la Tabla de Composición de Alimentos de Latinoamérica (10) incluye valores correspondientes al contenido de vitamina C en espinacas frescas crudas de varios países y sólo un dato de espinacas hervidas.

A partir de los antecedentes indicados, surgió la necesidad de determinar el contenido de vitamina C (AA y ADHA) en espinacas producidas en Argentina considerando los puntos de venta, los procesos industriales de conservación y los métodos de cocción habituales como posibles causas de pérdida del nutriente en el alimento.

\section{MATERIALES Y MÉTODO} Muestreo

Las muestras de espinaca fresca fueron adquiridas en establecimientos productores de hortalizas (quintas), comercios minoristas de verduras, hortalizas y frutas (verdulerías) y supermercados. Los productos supercongelados y en conserva se adquirieron en supermercados minoristas de la Provincia de Buenos Aires. En la selección de las espinacas supercongeladas se tuvieron en cuenta a aquellas de mayor presencia en el mercado regional. Las muestras de espinacas en conserva corresponden a la única marca disponible en los centros comerciales donde se realizó el muestreo.

\section{Preparación de las muestras}

Espinacas frescas: fueron analizadas en el día en que se adquirieron, conservadas bajo refrigeración y preservadas de la luz hasta el momento de procesamiento. Se lavaron con agua destilada, se escurrieron y secaron con papel adsorbente.

Inmediatamente se pesaron distintas fracciones a analizar:

Cruda. Una porción entre 40 y $50 \mathrm{~g}$ se homogeneizó con una procesadora doméstica en $75 \mathrm{~mL}$ de $\mathrm{HPO}_{3}$ $0.85 \mathrm{~g} / \mathrm{dL}$.

Sometida a hervor. Una porción de aproximadamente 100 g se cubrió con agua y se sometió a hervor durante tres minutos, luego de escurrida y a temperatura ambiente, se pesó y finalmente se procesó de la misma manera que la espinaca cruda.

Microondas. Otra porción de aproximadamente 100 $\mathrm{g}$ fue sometida a cocción en un horno de microondas doméstico, en un recipiente apropiado con agregado de $10 \mathrm{~mL}$ de agua, a potencia máxima $(1000 \mathrm{~W})$ durante 1 minuto, transcurrido este tiempo el proceso continuó como en el caso anterior.

Vapor. Otra porción de aproximadamente $100 \mathrm{~g}$ fue sometida a cocción al vapor durante 3 minutos a partir del inicio de la ebullición, en un recipiente de uso doméstico para este fin. Luego se trató de idéntica manera que en los casos anteriores.

Espinacas supercongeladas: se conservaron en freezer $\left(-18^{\circ} \mathrm{C}\right)$ hasta su procesamiento. Cada muestra se dividió en tres porciones: la primera, de aproximadamente $100 \mathrm{~g}$ se analizó sin ser sometida a cocción. La segunda de aproximadamente $150 \mathrm{~g}$ fue cubierta de agua y hervida durante 3 minutos y la tercera de aproximadamente $150 \mathrm{~g}$ fue sometida a cocción por microondas dentro del envase comercial o recipiente para microondas a potencia máxima durante 6 minutos. Finalmente cada porción fue triturada con una procesadora doméstica en $75 \mathrm{~mL}$ de HPO3 $0.85 \mathrm{~g} / 100 \mathrm{~mL}$.

Espinacas en conserva fueron escurridas y procesadas en idénticas condiciones que los casos anteriores, sin someterlas a ningún tratamiento previo de cocción.

Los homogenados resultantes de las preparaciones previas de todas las muestras fueron centrifugados a 4000 rpm durante 40 minutos en centrífuga refrigerada $\left(4^{\circ} \mathrm{C}\right)$ y las determinaciones se realizaron en el sobrenadante.

La masa de las muestras sometidas a cocción fue determinada antes y después del proceso con el fin de determinar los correspondientes factores de recuperación.

\section{Método analítico}

Las determinaciones de vitamina $\mathrm{C}$ se efectuaron por cromatografía líquida de alta resolución (HPLC), 
según el método de Berhens y Madère modificado (11).

Sobre una alícuota de cada muestra se determinó AA. A $4.00 \mathrm{~mL}$ del sobrenadante límpido se agregaron $1.15 \mathrm{~mL}$ de Buffer fosfatos $\mathrm{pH} 9.8$ y se completó el volumen hasta $10.00 \mathrm{~mL}$ con $\mathrm{HPO}_{3} 0.85 \mathrm{~g} / 100 \mathrm{~mL}, 0.5 \mathrm{~mL}$ de esta solución se diluyeron a $10.00 \mathrm{~mL}$ con solución de acetato de sodio $80 \mathrm{mM}$ : metanol (85:15) pH: 4.8 . La solución se filtró utilizando filtros de membrana de nylon $0.45 \mu$ y se analizó por HPLC.

Sobre una segunda porción se determinó en forma conjunta AA + ADHA. A $4.00 \mathrm{~mL}$ del sobrenadante límpido se agregó $1.15 \mathrm{~mL}$ de solución de ditiotreitol $0.5 \%$ $\mathrm{g} / \mathrm{dL}$ en buffer fosfatos $\mathrm{pH} 9.8$, se incubó 30 minutos a temperatura ambiente y luego se procedió de igual forma que para la terminación de AA.

Se utilizó un equipo de HPLC marca Könik con detector UV y software Clarity, Data Apex, con una columna RP C18 $250 \mathrm{~mm}$ por $4.6 \mathrm{~mm}$ (Varian, microsorb-MB) y fase móvil acetato de sodio $80 \mathrm{mM}$ y ácido metafosfórico $1.85 \mathrm{Mm} /$ metanol (85/15) pH: 4.6. La detección se realizó a $254 \mathrm{~nm}$.

La cuantificación se realizó utilizando como estándar externo ácido ascórbico Merck p.a disuelto en HPO3 $0.85 \mathrm{~g} / \mathrm{dL}$ y tratado con el mismo reductor que las muestras.

\section{Cálculo del factor de retención}

Se calculó el \% de retención utilizando la fórmula de Murphy E. $(12,22)$.

Retención de nutriente $(\%)=$

Contenido de nutriente/100 g de alimento cocido. $\mathrm{d}$

Contenido de nutriente/ $100 \mathrm{~g}$ de alimento crudo

Rendimiento porcentual en peso=

$\mathrm{d}=$ masa de alimento cocido. 100

masa de alimento crudo

\section{Análisis estadístico}

Cada muestra se analizó por duplicado y se informó el valor promedio. Sólo se consideraron aquellos resultados en los cuales los valores duplicados diferían en menos del $10 \%$. Para comparar el contenido de vitamina $\mathrm{C}$ en las muestras de espinacas frescas y supercongeladas, con y sin tratamiento culinario, se realizó el análisis de varianzas. Dado que los datos no cumplían con los supuestos de homogeneidad de varianzas y normalidad, se empleó la transformación de Box and Cox (((yij^0.5)-1)/0.5). La homogeneidad de varianzas se verificó utilizando el test de Levene ( $p>0.05$ ) y para comparar las medias se utilizó el mé- todo de Bonferroni. En todos los cálculos se utilizó el software estadístico R (13).

\section{RESULTADOS Y DISCUSIÓN}

En la espinaca fresca cruda, el contenido de vitamina C resultó ser ampliamente variable tanto para la vitamina total (AA+ADHA) como para la relación AA / ADHA (tabla 1). El contenido promedio de AA representa cerca del $70 \%$ del total de vitamina con valores comprendidos entre 26 y $100 \%$. Esta dispersión es atribuible a diversos factores como diferencias genotípicas, agronómicas, de manejo poscosecha y la falta de homogeneidad propia del vegetal.

Los valores determinados por otros autores y por diferentes metodologías analíticas, para el mismo vegetal resultaron ser ampliamente variables; Duodu y colaboradores (14) utilizaron la titulación con 2,6 dicloro fenol-indofenol e informaron valores de AA de $14 \pm 2.6 \mathrm{mg} / 100 \mathrm{~g}$, Favell (9) utilizó una variante del mismo método y los valores de AA y vitamina C resultaron 22 y $31 \mathrm{mg} / 100 \mathrm{~g}$ respectivamente. La utilización de esta metodología se debe limitar a muestras donde el analito se encuentra libre de la presencia de sustancias oxidables o coloreadas que generan resultados falsamente incrementados o perturban la detección del punto final respectivamente (23). Ozkan y colaboradores (15) determinaron la absorbancia de extractos en ácido oxálico a $520 \mathrm{~nm}$ y reportaron un contenido de AA de $50.18 \pm 1.36 \mathrm{mg} / 100 \mathrm{~g}$. Debido a que esta determinación se realiza a un valor de longitud de onda en el que muchos compuestos presentes en las matrices alimentarias contribuyen a la absorbancia, es necesario el pleno conocimiento de la naturaleza de la muestra a analizar antes de la implementación de esta técnica. Además esta metodología solo determina AA y no ADHA.

Nuestros resultados son cercanos a los informados en la base de datos del USDA (16) que presenta valores de vitamina $C$ de $28.1 \pm 4.129 \mathrm{mg} / 100 \mathrm{~g}$ y fueron obtenidos por titulación con 2,6 dicloro fenol-indofenol o por el método fluorométrico. Este último método, reconocido por la Association Oficial of Analytical Chemistry (AOAC), presenta elevada sensibilidad pero requiere una corrección basada en la altura de pico de blancos y un estricto control de $\mathrm{pH}$.

Al evaluar los resultados según el sitio de venta (tabla 2) se pudo observar que las mayores concentraciones de vitamina $\mathrm{C}$ se hallaron en aquellas muestras adquiridas en establecimientos productores de hortalizas (quintas) y en supermercados, donde el producto fresco esta conservado a bajas temperaturas. Los valores más bajos se observaron en las espinacas adquiridas en verdulerías, donde las hojas se conservan a temperatura 
ambiente. Esto coincide con investigaciones anteriores (9) que determinaron una rápida pérdida de vitamina $\mathrm{C}$ durante el almacenamiento de los vegetales en distintas condiciones, con un contenido residual del $10 \%$ luego de tres días a temperatura ambiente y del $20 \%$, luego de 1 semana de almacenamiento refrigerado.

En cuanto al contenido de ADHA, el valor más elevado se encontró en muestras adquiridas en supermercados, demostrando un mayor grado de avance del deterioro de la vitamina respecto de las muestras adquiridas en quintas. Sin embargo el valor más bajo de ADHA corresponde a las muestras provenientes de verdulerías, que en concomitancia con el bajo contenido de vitamina indicaría la degradación irreversible de la misma.

En el hervor se obtuvieron los menores valores de retención de vitamina (tabla 3), incluso en algunas muestras se detectaron pérdidas de hasta el $100 \%$. Estos resultados son atribuibles principalmente a pérdidas por solubilidad en el agua utilizada para la cocción. Esto resulta coincidente con los resultados presentados por Somsub (2) que informó que el contenido de vitamina $\mathrm{C}$ en vegetales de Thailandia es significativamente reducido por el hervor.

En la cocción por microondas y al vapor no se obser-

\section{TABLA 1}

\section{Contenido vitamina $\mathbf{C}$ y sus formas activas en espinacas frescas $\mathbf{y}$ procesadas}

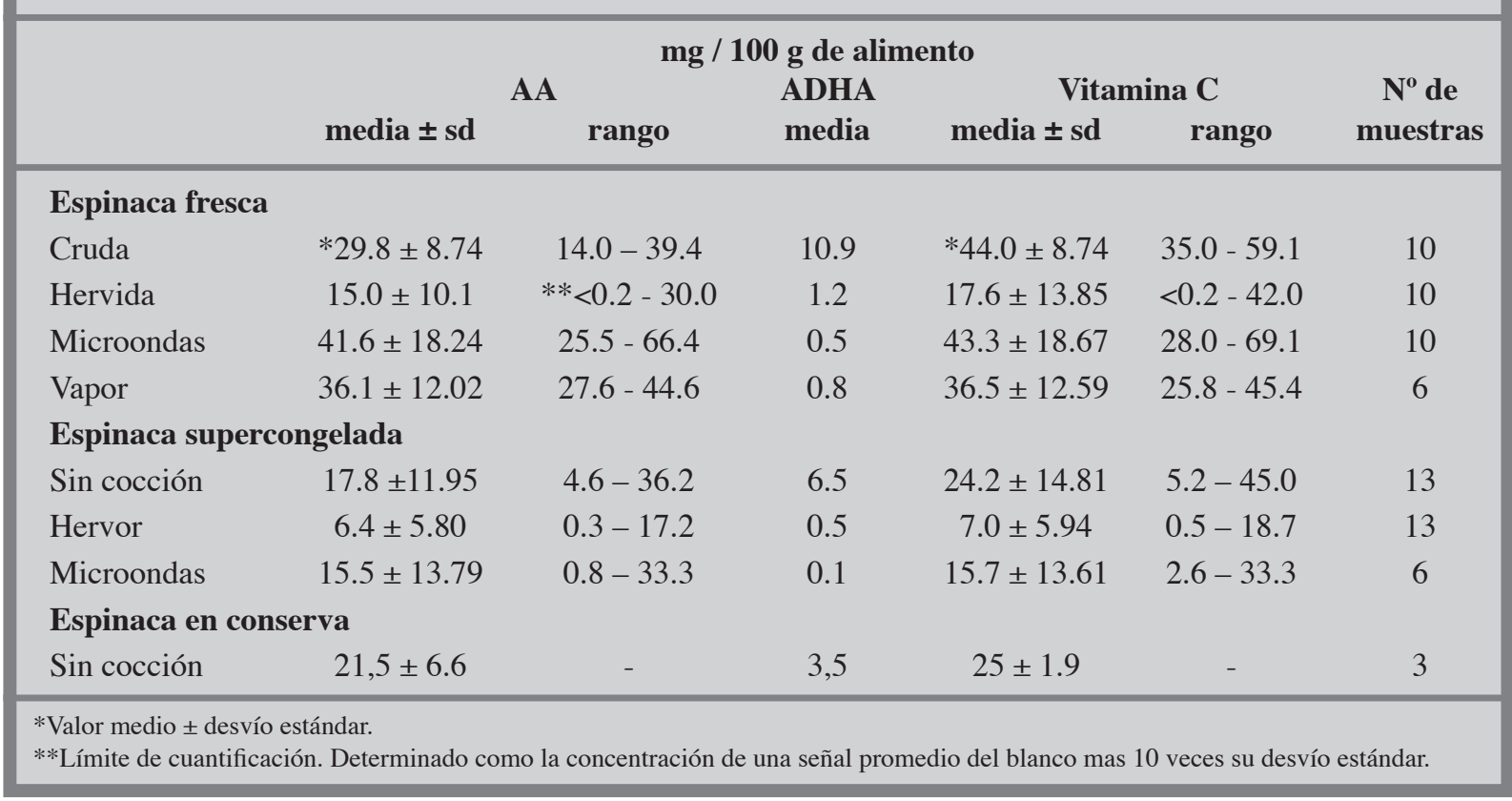

\section{TABLA 2}

\section{Contenido de vitamina $\mathrm{C}$ en espinacas frescas según punto de comercialización}

\begin{tabular}{|c|c|c|c|}
\hline & $\mathbf{A A}$ & $\begin{array}{c}\text { mg / } 100 \text { g de alimento } \\
\text { Vitamina C (AA+ ADHA) }\end{array}$ & ADHA \\
\hline Quinta de hortalizas & $* 36,5 \pm 4,82$ & $48,2 \pm 12.10$ & $* * 11,7$ \\
\hline Verdulerías & $29,8 \pm 9,00$ & $36,8 \pm 1.70$ & 7,0 \\
\hline Supermercados & $23,3 \pm 8,33$ & $49,4 \pm 4.13$ & 26,1 \\
\hline
\end{tabular}


varon diferencias significativas respecto del contenido de vitamina $\mathrm{C}$ de las espinacas frescas crudas, aunque puede detectarse una disminución del contenido de ADHA. Además, en algunas muestras cocidas por microondas se obtuvieron FR\% (tabla 3) que superaron el $100 \%$. Esto podría deberse a cambios que ocurren durante la cocción como la inactivación de enzimas, con efecto deletéreo sobre la vitamina y a daños tisulares en el vegetal que favorecerían la extracción del ácido ascórbico durante la preparación de la muestra analítica.

Otros autores (17 - 18) estudiaron el efecto de distintos métodos de cocción en cassava y encontraron que en la cocción por microondas se obtienen las mayores retenciones de esta vitamina. Además, detectaron pérdidas importantes de vitamina $\mathrm{C}$ en la cocción al vapor, resultado que se contrapone con el obtenido en nuestro laboratorio. Esto podría deberse a tiempos de cocción muy superiores (1 hora) respecto de los utilizados en espinaca (3 minutos).

El contenido de vitamina $C$ en espinaca supercongelada se encuentra sustancialmente disminuido respecto al vegetal fresco (tabla 1). Aquí es necesario tener en cuenta que la conservación por congelación incluye un escaldado o blanqueo para lograr inactivación enzimática, seguido de un congelado rápido a $-18{ }^{\circ} \mathrm{C}$ (supercongelado) (20). Negi (19) demostró que el blanqueo no sólo modifica las características sensoriales del producto sino que además provoca pérdidas considerables de nutrientes como la vitamina $\mathrm{C}$. También debe contemplarse una posible pérdida a consecuencia del proceso de descongelación anterior a la preparación culinaria. Asimismo la dispersión de los valores es mayor que en el caso de las verduras frescas, esto es consecuencia del contenido inicial de la vitamina al momento del procesamiento, de las diferentes condiciones aplicadas en los procesos tecnológicos para obtener el producto congelado y de la conservación adecuada por mantenimiento de la cadena de frío del producto al momento de ser consumido (9-19).

La cocción de las espinacas supercongeladas disminuyó aún más el contenido de vitamina C. La retención es mayor en el caso de la cocción por microondas que en el caso del hervor donde los valores disminuyen marcadamente (tabla 1).

A diferencia de las espinacas frescas cocidas por microondas, en las espinacas supercongeladas se observa disminución significativa del contenido total de vitamina respecto del producto crudo. Esto se debe a que el tiempo de cocción del producto congelado es superior al empleado para las espinacas frescas.

Luego de la cocción de las espinacas cruda y supercongelada por los distintos métodos de cocción, se observa una disminución del contenido porcentual de ADHA respecto de las formas no cocidas. Dependiendo del método de cocción utilizado, estas pérdidas serían atribuibles a las diferencias de solubilidad de ambas formas activas y sobre todo a la degradación irreversible del ADHA a formas sin actividad vitamínica.

En el caso de las espinacas en conserva (tabla 1), el contenido de vitamina $\mathrm{C}$ es algo inferior al de las hojas frescas. Aquí no se evaluaron los métodos de cocción debido a que la elaboración de la conserva que incluye escaldado y esterilización, reemplaza la cocción previa a la preparación culinaria. Debido a la elaboración y conservación del producto en un medio líquido, es de esperar que el contenido de vitamina $\mathrm{C}$ sea inferior al encontrado, pero en nuestro país el código alimentario permite el agregado de ácido 1- ascórbico o eritórbico a las conservas hasta $500 \mathrm{mg} / \mathrm{Kg}$ como antioxidante sin ser declarado en el rótulo (21), incorporándose así otra

\section{TABLA 3}

Factores de retención de AA y vitamina C en espinacas sometidas a cocción.

\section{AA (\%) Vitamina C (\%) \\ Factores de retención}

\section{Espinaca fresca}

Hervor

Microondas

vapor

Espinaca supercongelada

Hervor

Microondas
$26 \pm 23$

$100 \pm 34$

$91 \pm 30$

$27 \pm 15$

$67 \pm 31$
$19 \pm 18$

$74 \pm 22$

$80 \pm 30$ 
causa de variabilidad en el contenido de vitamina en los diferentes productos comercializados.

\section{CONCLUSIONES}

Los resultados obtenidos en las determinaciones de ácido ascórbico por HPLC en espinacas frescas crudas y hervidas resultaron comparables a los obtenidos por otros investigadores aún partiendo de metodologías analíticas diferentes (tabla 1).

El contenido de vitamina luego de la preparación es dependiente de la metodología de cocción y los parámetros del proceso utilizado. La cocción por microondas y vapor permitió mayor retención del nutriente respecto del hervor.

Los métodos de conservación y de cocción aplicados incrementaron aún más la variabilidad en el contenido de vitamina respecto al vegetal fresco crudo, y es esperable que las preparaciones culinarias habituales aumenten dicha variabilidad debido a la falta de estandarización de las condiciones del proceso utilizado. Esto nos permite concluir que no es posible caracterizar a la espinaca (Spinacea oleracea L y sus variedades) como una fuente cuantitativamente confiable de vitamina $\mathrm{C}$.

Debe destacarse la importancia de utilizar una técnica analítica que determine el contenido total de la vitamina debido a las elevadas concentraciones de ADHA que se encontraron en algunas muestras analizadas.

Finalmente, las determinaciones realizadas permiten contar con información actualizada correspondiente al contenido de vitamina $\mathrm{C}$ y sus formas activas en espinaca fresca y procesada sometidas a las formas más habituales de cocción.

\section{RESUMEN}

La labilidad de la vitamina $\mathrm{C}$, las nuevas variables de cultivo, manejo y conservación postcosecha de los vegetales y los tratamientos culinarios previos a su consumo pueden provocar modificaciones en el contenido de este nutriente. Para contar con información actualizada y propia del contenido de vitamina $\mathrm{C}$ en alimentos frescos y procesados, se determinó este nutriente en espinacas frescas y sometidas a los métodos de conservación actuales considerando también, la influencia de los sitios de expendio y la aplicación de los tratamientos culinarios más comunes. La determinación de vitamina $\mathrm{C}$, constituida por ácido ascórbico y dehidroascórbico, se realizó empleando HPLC. Los valores medios de vitamina en espinacas sin procesar y su desvío estándar son 44.0 y $8,74 \mathrm{mg} / 100 \mathrm{~g}$, en productos supercongelados 24,2 y $14,81 \mathrm{mg} / 100 \mathrm{~g}$ y en conservas de 25.0 y $1.9 \mathrm{mg} / 100 \mathrm{~g}$. Esta variabilidad depende de factores genotípicos y condiciones de conservación. La cocción por microondas y vapor generaron las mayores retenciones del nutriente, mientras que el hervor provocó grandes pérdidas de ambas formas vitamínicas.

Palabras clave: Espinaca; vitamina C; ácido ascórbico; ácido dehidroascórbico; HPLC.

Dirigir la correspondencia a:

Profesor

Andrés Fabián Pighín G.

Departamento de Ciencias Básicas

Universidad Nacional de Luján

Buenos Aires, Argentina

Av. Constitución y Ruta Nacional No 5, Luján (6700)

Teléfono: +54 (02323) 423979 / 423171

Fax: +54(02323) 425795

Email:analitic@mail.unlu.edu.an

Agradecimientos: Este trabajo fue financiado por el Departamento de Tecnología de la Universidad Nacional de Luján. Agradecemos a la Ingeniero María Laura Vranik, al Dr. José Manuel Aguirre y la Licenciada María Cristina de Landeta por su colaboración en el desarrollo de este trabajo.

\section{BIBLIOGRAFÍA}

1. Panel on Dietary Antioxidants and Related Compounds, Subcommittees on Upper Reference Levels of Nutrients and Interpretation and Uses of DRIs, Standing Committee on the Scientific Evaluation of Dietary Reference Intakes, Food and Nutrition Board. Dietary Reference Intakes for Vitamin C, Vitamin E, Selenium, and Carotenoids. Food and Nutrition Board Institute of Medicine. NATIONAL ACADEMY PRESS Washington, D.C. ISBN: 0-309-59719-6. (2000).

2. Somsub W. Kongkachuicai R. Sungpuag P. Charoensiri R. Effects of three conventional cooking methods on vitamin $\mathrm{C}$, tannin, myo-inositol phosphates contents in select Thai vegetables, J Food Composition Analysis 2008; 21: 187-197.

3. Kiyota M. Numayama N. Goto, K. Circadian rhitms of the L-ascorbic acid level in Euglena and spinach, $\mathrm{J}$ of Photochemistry and photobiology B: Biology 2006; 84: 197-203.

4. Potters G. De Gara L. Asard H. Horemans N. Ascorbate and glutathione: guardians of the cell cycle, partners in crime?, Plant Physiology Biochem 2002; 40: 537-548.

5. Toledo M. Ueda Y. Imahori Y. Ayaki M.. L- ascorbic acid metabolism in spinach (Spinacia oleracea L) during postharvest stage in Light and dark. Postharvest, Biol Technol 2003; 28(1): 47-57. 
6. Linder M. Food Quality and its determinants, from field to table: Growing Food its Storage and preparation, 2nd edition.Ed MC Linder Elsevier, New York, Amsterdam, London, Tokyo. "Nutrition and prevention of Chronic Diseases". Technical Report Series N ${ }^{\circ} 797$. WHO, Geneve. 1991:329-348.11.

7. Giannakourou M. Taoukis P. Kinetic modeling of vitamin $\mathrm{C}$ loss frozen green vegetables under variable storage conditions, Food Chem 2003; 83: 33-41.

8. Sauberlich H. Ascorbic Acid. Present Knowledge in nutrition, 6th Ed. International Science Institute. Nutrition Fundation. Washington D.C.1990; pp. 132-141.

9. Favell D. A comparison of the vitamin C content of fresh and frozen vegetables, Food Chem 1998; 62 (1): 59-64.

10. “FAO / LATINFOODS. 2002. Tabla de Composición de Alimentos de_América Latina". (http:// www.rlc.fao.org/bases/alimento).

11. Behrens W. Madère R. Highly Sensitive High Performance Liquid Chromatography Method for the estimation of Ascorbic and Dehidroascorbic Acid Tissues, Biological Fluids and Foods, Anal Biochem 1987;165: 102-107.

12. Murphy E. Criner P. Gray B. Comparisons of methods for calculating of nutrient in cooked foods, $\mathrm{J}$ Agricul Food Chem1975; 23(6). 1153- 1157

13. Software estadístico R (http://cran.r-project.org).

14. Duodu K. Minnar A. Taylor J. Effect of cooking and irradiation on fhe labile vitamins and antinutrient content of a tradicional African sorghunporridge and spinach relish, Food Chem 1999; 66 (1): 21-27.
15. Ozkan Alibas I. Akbudak B. Akbudak N. Microwave drying characteristcs of spinach, J Food Engeneering 2007; 78: 577-583.

16. United States department of agriculture (USDA). National Nutrient Database for Standard Reference Release 18 yww.nal.usda.gov/fnic/foodcomp/ Data/SR18/sr18).

17. Kumar S. Aalbersberg. Nutrient retention in foods after earth-oven cooking compared to other forms of domestic cooking 2. Vitamins, J Food Composition Analysis 2006; 19: 311-320.

18. Kumar S. Aalbersberg. Nutrient retention in foods after earth-oven cooking compared to other forms of domestic cooking 1. Proximates, carbohydrates and dietary fibre, J Food Composition Analysis 2006, 19: 302-310.

19. Negi, P. Roy, S. Effect of blanching and drying methods on $\beta$-carotene, ascorbic acid and chlorophyll retention of leafy vegetables, Lebnsm.-Wiss. U.-Technol.2006; 33: 295-298

20. Código Alimentario Argentino Artículos 162 y 163 www anmat.gov.ar/codigoa/Capitulo_III_Vegetales_2007-05.

21. Código Alimentario Argentino Artículos 926 y 932 www anmat.gov.ar/codigoa/Capitulo_XI_Vegetales_2007-05.

22. Bognár A. Piekarski J. Guidelines for recipe information and calculation of nutrient composition of prepared foods (Dishes), J Food Composition Analysis 2000;13: 391-411.

23. Arya S. Mahajan M. Jain P. Non-espectrophotometric methods for the determination of Vitamin C. Analytica Chem Acta 2000;417:1-14. 